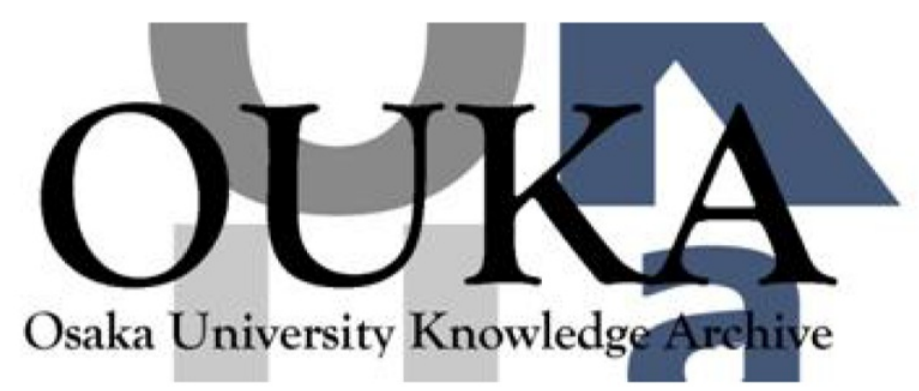

\begin{tabular}{|c|l|}
\hline Title & $\begin{array}{l}\text { Nanometer-sized gelatin particles prepared by } \\
\text { means of gamma-ray irradiation }\end{array}$ \\
\hline Author(s) & $\begin{array}{l}\text { Furusawa, Kazuya; Nagasawa, Naotsugu; Yoshi i, } \\
\text { Fumio et al. }\end{array}$ \\
\hline Citation & Colloid and Polymer Science. 283(2) p. 229-p. 233 \\
\hline Issue Date & $2004-12$ \\
\hline oaire:version & AM \\
\hline URL & https://hdl. handle. net/11094/25930 \\
\hline rights & \\
\hline Note & \\
\hline
\end{tabular}

Osaka University Knowledge Archive : OUKA

https://ir. Library. osaka-u. ac. jp/

Osaka University 


\section{Nanometer-sized gelatin particles prepared by means of}

\section{gamma-ray irradiation}

Kazuya Furusawa, ${ }^{\text {a Ken Terao, },{ }^{\mathrm{a}} \text { * }}$ Naotsugu Nagasawa, ${ }^{\mathrm{b}}$ Fumio Yoshii, ${ }^{\mathrm{b}}$ Kenji Kubota, ${ }^{\mathrm{a}}$ and Toshiaki Dobashi ${ }^{\mathrm{a}}$

${ }^{a}$ Department of Biological and Chemical Engineering, Faculty of Engineering, Gunma University, 1-5-1, Tenjin-cho, Kiryu, Gunma 376-8515, Japan

${ }^{\mathrm{b}}$ Takasaki Radiation Research Establishment, Japan Atomic Energy Research Institute, Watanuki-machi, Takasaki, Gunma 370-1292, Japan.

* Corresponding author. Phone: +81-277-30-1478, Fax: +81-277-30-1401

E-mail address: terao@bce.gunma-u.ac.jp (K. Terao) 


\begin{abstract}
Nanometer-sized gelatin particles have been prepared by means of gamma-ray irradiation and characterized by static and dynamic light scattering combined with circular dichroism measurements. The molecular weight of the nanoparticles was much larger than that of the original gelatin molecules, whereas the hydrodynamic radius was much smaller. Radius of gyration evaluated from the angular dependence of the static light scattering intensity decreased with increasing irradiation dose. Circular dichroism spectra of the gelatin nanoparticles were independent of temperature, and it is suggested that the nanoparticles consist of highly and randomly packed gelatin and their conformation is stable against the temperature change.
\end{abstract}

Keywords: gelatin, gamma-ray, crosslinking, nanogel, light scattering 


\section{INTRODUCTION}

Nanometer-sized capsules and spheres have attracted growing interests as drug delivery carriers for the treatment of various diseases including cancer and infections and also as an essential part in BioMEMS [1,2]. The most important requirement for such ultra fine particles is biosafety, high stability and controlled functional properties. Gelatin has been used as a starting material in a variety of applications because of its biosafety verified in food and clinical fields for a long time [3]. Aqueous gelatin solution undergoes gel to sol transition around $20-30^{\circ} \mathrm{C}$ depending on the concentration. To avoid the transformation to sol for the use of a drug delivery carrier, various methods such as chemical treatments and electromagnetic wave irradiation have been developed [4].

Recently, in the course of gamma-ray and electron beam irradiation to gelatin aqueous solutions, we observed that the light scattering intensity of dilute solutions of the irradiated gelatin was significantly stronger than that before the irradiation [5]. The scattered light intensity did not decrease even after the solution was heated up to $60^{\circ} \mathrm{C}$. These results suggest that particles with high molecular weight grow in the solution during the irradiation process. Since no particles could be observed under an optical microscopy, the size of the particles should be quite less than the wavelength of visible light. Such a phenomenon has been only observed for a limited number of synthetic polymers [6]. The gelatin particles prepared by such high-energy electromagnetic waves have advantages compared with those manufactured from emulsion [4(a),7] because a large amount of organic solvents is needed to prepare the emulsion. It is very interesting to characterize the gelatin nanoparticles or swollen nanogel, and to find the appropriate conditions to control the 
structure of the particles. From this motivation, we have carried out static and dynamic light scattering combined with circular dichroism (CD) measurements for aqueous gelatin solutions with various concentrations and irradiation doses.

\section{EXPERIMENTAL}

Preparation of Microcapsules

Gelatin from porcine skin (Nitta gelatin APH-250, lot No. 030203, Bloom value is 250) was purified by reprecipitation from water into ethanol. Three aqueous solutions with the concentrations $c$ being $1.0 \times 10^{-3}, 5.0 \times 10^{-4}$ and $2.5 \times 10^{-4} \mathrm{~g} \mathrm{~cm}^{-3}$ (coded as G1, G2 and G3) were prepared at $40^{\circ} \mathrm{C}$. After each solution was filtrated through a membrane filter made of cellulose acetate (pore size $0.8 \mu \mathrm{m}$ ), it was centrifuged at 9,000 $\times \mathrm{g}$ and then the middle portion was transferred carefully into a quartz cell with a pipette in order to purify it optically for the light scattering measurements. All the samples were stored at about $5^{\circ} \mathrm{C}$ until the gamma-ray irradiation. The gelatin solutions were irradiated at room temperature around $20^{\circ} \mathrm{C}$ with gamma-ray generated from ${ }^{60}$ Co source (Takasaki Radiation Chemistry Research Establishment) at a dose rate of $10 \mathrm{kGy} / \mathrm{h}$. The samples thus obtained were designated as shown in Table 1. Appreciable precipitations were observed in the samples G3-0, G3-10, G3-20 and G2-20 after the irradiation.

\section{Characterization Techniques}

A laboratory made light scattering photometer and ALV 5000/E, multiple-tau digital correlator, were used for the static and dynamic light scattering measurements 
[8]. A vertically polarized He-Ne laser operated at the wavelength $\lambda_{0}$ of $632.8 \mathrm{~nm}$ was used as the incident beam, and the vertically polarized scattered light was detected over the range of the scattering angle $\theta$ from 30 to $130^{\circ}$, corresponding to $0.007 \sim 0.026 \mathrm{~nm}^{-1}$ in the magnitude of scattering vector $q\left[\equiv\left(4 \pi n / \lambda_{0}\right) \sin (\theta / 2)\right]$ with $n$ being the refractive index of the solvent. Light scattering measurements were carried out at $40^{\circ} \mathrm{C}$ for the samples in which no precipitations were observed. The specific refractive index increment $\partial \mathrm{n} / \partial \mathrm{c}$ was estimated to be $0.184 \mathrm{~cm}^{3} \mathrm{~g}^{-1}$ from the plot of $\partial n / \partial c$ vs. $\lambda_{0}{ }^{-2}$ of literature values [9]. CD measurements at 20, 30 and $40^{\circ} \mathrm{C}$ were performed by JASCO-J720W circular dichroism spectropolarimeter using a quartz cell of $1 \mathrm{~cm}$ pass length at the wavelength of the incident light between 190 and $250 \mathrm{~nm}$.

A conventional Zimm plot was employed to obtain the weight averaged molecular weight and the radius of gyration. The normalized electric field correlation function $g^{1}(t)$ was obtained from the autocorrelation function of scattered light intensity, and was analyzed by the CONTIN method. $g^{1}(t)$ is expressed formally by the distribution function $G(\tau)$ of the characteristic time $\tau$ as

$$
g^{1}(t)=G(\tau) \exp (-t / \tau) \mathrm{d} \tau
$$

where $\tau$ relates to the hydrodynamic radius of the particle, $R_{\mathrm{h}}$, as $\tau=\left(6 \pi \eta_{0} R_{\mathrm{h}} / k_{\mathrm{B}} T\right) / q^{2}$ with $\eta_{0}$ being the solvent viscosity. Eq. 1 is rewritten more conveniently as

$$
g^{1}(t)=\tau G(\tau) \exp (-t / \tau) \mathrm{d}(\log \tau)=A\left(R_{\mathrm{h}}\right) \exp (-t / \tau) \mathrm{d}\left(\log R_{\mathrm{h}}\right)
$$


That is, $A\left(R_{\mathrm{h}}\right)$ means the distribution of hydrodynamic radius and the fraction of intensity having the hydrodynamic radius of $R_{\mathrm{h}}$ relative to the total scattered intensity. Therefore, in case that $A\left(R_{\mathrm{h}}\right)$ has the bimodal distribution, intensities of the respective modes can be evaluated from the integration of $A\left(R_{\mathrm{h}}\right)$ corresponding to the respective mode. In fact, Jinbo et al. succeeded to estimate the structure factor of aggregates of polymer molecules in the solution by this procedure [10].

\section{RESULTS AND DISCUSSION}

\section{Molecular Weight and Hydrodynamic Radius}

Figure 1 illustrates the angular dependence of the inverse scattering intensity $K c / R_{\theta}$, where $K$ and $R_{\theta}$ are the optical constant and the reduced scattering intensity at $\theta$, respectively. Apparent molecular weights $M_{\text {app }}$ were evaluated from the intercept of each curve drawn to represent the experimental data and are listed in Table 1. $M_{\text {app }}$ values for the sample without irradiation, G1-0 and G2-0, are considerably larger than the molecular weight $3 \times 10^{5}$ measured for gelatin samples with a larger bloom value in formamide [11]. This suggests a substantial coexistence of gelatin aggregates in water. With increasing irradiation dose, $M_{\text {app }}$ increases whereas the slope of the angular dependence curve decreases distinctly. On the other hand, the results from the dynamic light scattering measurements are shown in Fig. 2. For the samples without irradiation, G1-0 and G2-0 (filled circles), a larger peak (slow mode) around $300 \mathrm{~nm}$ and a smaller peak (fast mode) at less than $10 \mathrm{~nm}$ are observed. With increasing dose, the relative intensity of the smaller peak increases and that of the larger peak decreases. Considering the increase of static scattering intensity with 
increasing dose as shown in Fig. 1, it is suggested that nanogels with high molecular weight and the hydrodynamic radius of ca. $10 \mathrm{~nm}$ grow in the solution, while the original aggregates disintegrate, by the gamma-ray irradiation. The hydrodynamic radii $R_{\mathrm{h}, 1}$ and $R_{\mathrm{h}, 2}$ estimated from the peaks for the slow and fast modes are summarized in Table 2. As shown typically in $R_{\mathrm{h}, 2}$ for G1-20, the size and the molecular weight of nanogels increase with increasing dose, about 2.6 times larger than that of G1-10.

Because the ratio of the peak area in Fig. 2 corresponds to the ratio of the scattering intensity, $R_{\theta}$ for each component can be estimated. Fig. 3 shows the plot of angular dependence of the reciprocal $R_{\theta}$, estimated from the results for the fast mode with smaller size. The slopes are much smaller than those for the (total) scattering intensity in Fig. 1, and the apparent radius of gyration is smaller than $20 \mathrm{~nm}$ considering the experimental uncertainty in the intensity $R_{\theta}$ thus evaluated. This fact is consistent with the results of the CONTIN analysis described above.

The action of gamma-ray on most polymers in dilute aqueous solutions occurs mainly through an indirect effect: most part of radiation energy is absorbed by water, hydroxyl radicals and hydrogen atoms are yielded, and they react rapidly with polymers [6]. Both $M_{\mathrm{app}}$ and $R_{\mathrm{h} 2}$ observed for G2 $\left(c=5.0 \times 10^{-4}\right)$ are larger than the corresponding ones for G1 $\left(c=1.0 \times 10^{-3}\right)$. The higher efficiency of the lower gelatin concentration sample to form nanoparticles is consistent with the predominance of the indirect effect. The increase of the fast mode and the corresponding decrease of the slow mode resulted from irradiation suggests that the source of the nanoparticles with large molecular weight is the originally aggregated gelatin particles. In the radiation process, the high-energy gamma ray produce many 
radicals simultaneously inside the aggregates, and scission and crosslinking inside each aggregate occurs to form nanoparticles, since the diffusion of the aggregates is too slow to react between different aggregates.

\section{Helical Structure}

Helix content (proline-rich collagen-type triple helix) of gelatin molecules depends significantly on the temperature, which is closely related to the sol-gel transition [12]. Fig. 4 illustrates the circular dichroism spectra for aqueous gelatin solution with and without irradiation. Characteristics of observed spectra at $20^{\circ} \mathrm{C}$ in (A) with negative peak around $190 \mathrm{~nm}$ and positive peak around $220 \mathrm{~nm}$ could be attributed to the mixed contributions of random coil conformation and collagen-type triple helix, and the smearing of the curve with increasing temperature corresponds to the gel to sol phase transition. CD spectra of typical collagen-type triple helix show a negative peak around $200 \mathrm{~nm}$ and a positive peak around $220 \mathrm{~nm}$ [13]. With increasing temperature, triple helix should break (gel to sol transition occurs), resulting in the decrease of absolute magnitudes of positive and negative peaks, then the contribution from the random coil conformation becomes dominant. In contrast, the spectra for the irradiated gelatins are independent of temperature, and the intensity is much smaller than that for the unirradiated one as shown in (B) and (C). It is known that the conformational transition like the helix-coil transition becomes less clear as lowering the molecular weight [14]. Therefore, the results of circular dichroism might suggest that the molecular weight between the crosslinking points in the nanogels is too short to exhibit the temperature dependence of CD spectra, i.e., the conformational change cannot be realized, and the random coil conformation is 
prevailing. According to Fig. 2, the larger peak (slow mode) remains substantially at the irradiation dose of $10 \mathrm{kGy}$. Therefore, the contribution to the CD spectrum from the slow mode should not be ignored. However, the size of such a mode decreases significantly from the original one, and the chain length should become short enough so as not to constitute triple helix and/or aggregate with sufficient length and/or size. Of course, the present result is still preliminary. The circular dichroism per residue $[\Theta]$ is not analyzed yet because of the uncertainty of the constituent sequence of the nanogel. Further analyses of the detailed characterization of the nanogels thus prepared should be necessary.

\section{CONCLUSIONS}

In this work gelatin nanogels were newly prepared from aqueous gelatin solution by means of gamma-ray irradiation without any chemical reagents. Static and dynamic light scattering revealed that the nanoparticles have large molecular weight, while the hydrodynamic radius is as small as ca. $10 \mathrm{~nm}$. It was also suggested that the size of the nanoparticle could be controlled by adjusting the preparation condition of irradiation dose and gelatin concentration. According to the CD measurements, the ordered conformation of the original gelatin could be replaced by random conformation in the nanogel, and such a structure is stabilized. The highly and randomly packed structure granted a very high stability against the temperature change to the nanogel.

Acidic and basic gelatins adsorb basic and acidic polypeptides, respectively, and the complex compounds are utilized as a bioactive implant in tissue engineering [4(a)]. In a previous paper we confirmed that the selective adsorption properties are 
maintained in gelatin microbeads even after the irradiation as high as $60 \mathrm{kGy}$ dose. Furthermore, the biodegradability is controlled by the amount of irradiation dose [7]. These characteristics are expected also for the newly prepared nanogels. Since we can control the pore size (crosslinking density) and the particle size of the nanogels, it is quite interesting to investigate the potential use of the nanogels prepared by means of irradiation of gamma-ray as a candidate of such an intelligent BioMEMS.

\section{REFERENCES}

[1] P. Couvreur, G. Barratt, E. Fattal, P. Legrand and C. Vauthier, Crit. Rev. Ther. Drug Carrier Syst. 19 (2002) 99.

[2] D. A. LaVan, T. McGuire and R. Langer, Nature Biotechnology 21 (2003) 1184.

[3] D. Zekorn, Bibl Haematol. 33 (1969) 131.

[4] (a) Y. Tabata and Y. Ikada, Adv. Drug Delivery Rev. 31 (1998) 287. (b) S. Matsuda, N. Se, H. Iwata and Y. Ikada, Biomaterials 23 (2002) 2901.

[5] K. Terao, N. Nagasawa, H. Nishida, K. Furusawa, Y. Mori, F. Yoshii and T. Dobashi, J. Biomater. Sci. Polym. Ed. 14 (2003) 1197.

[6] (a) S. Kadlubowski, J. Grobelny, W. Olejniczak, M. Cichomski and P. Ulanski, Macromolecules 36 (2003) 2484. (b) P. Ulanski, I. Janik and J. M. Rosiak, Radiat. Phys. Chem. 52 (1998) 289.

[7] K. Terao, T. Karino, N. Nagasawa, F. Yoshii, M. Kubo and T. Dobashi, J. Appl. Polym. Sci. 91 (2004) 3083.

[8] D. Ito and K. Kubota, Macromolecules 30 (1997) 7828.

[9] T. M. McMeekin, M. L. Groves and N. J. Hipp, Advan. Chem. Ser. 44 (1964) 54. [10] Y. Jinbo, O. Teranuma, M. Kanao and T Sato, Macromolecules 36 (2003) 198. 
[11] C. Wu, Macromolecules 26 (1993) 5423.

[12] For example, C. Joly-Duhamel, D. Hellio and M. Djabourov, Langmuir 18 (2002) 7208.

[13] (a) T. Yoneyama, H. Kasuya, H. Onda, H. Akagawa, K. Hashiguchi, T. Nakajima, T. Hori and I. Inoue, Stroke 35 (2004) 443. (b) K. Ma, L.-S. Kan and K. Wang, Biochemistry 40 (2001) 3427.

[14] A. Teramoto, Prog. Polym. Sci. 26 (2001) 667. 
TABLE 1. $\quad M_{a p p}$ of Aqueous Gelatin with or without Irradiation.

\begin{tabular}{lccc}
\hline Sample & $c / \mathrm{g} \mathrm{cm}^{-3}$ & Dose / kGy & $M_{\text {app }}$ \\
\hline G1-0 & $1 \times 10^{-3}$ & 0 & $1.4 \times 10^{6}$ \\
G1-10 & $1 \times 10^{-3}$ & 10 & $1.7 \times 10^{6}$ \\
G1-20 & $1 \times 10^{-3}$ & 20 & $3.6 \times 10^{6}$ \\
G2-0 & $5 \times 10^{-4}$ & 0 & $1.5 \times 10^{6}$ \\
G2-10 & $5 \times 10^{-4}$ & 10 & $3.5 \times 10^{6}$ \\
G2-20 & $5 \times 10^{-4}$ & 20 & $\mathrm{~N} / \mathrm{A}^{\mathrm{a}}$ \\
G3-0 & $2.5 \times 10^{-4}$ & 0 & $\mathrm{~N} / \mathrm{A}^{\mathrm{a}}$ \\
G3-10 & $2.5 \times 10^{-4}$ & 10 & $\mathrm{~N} / \mathrm{A}^{\mathrm{a}}$ \\
G3-20 & $2.5 \times 10^{-4}$ & 20 & $\mathrm{~N} / \mathrm{A}^{\mathrm{a}}$ \\
\hline
\end{tabular}

${ }^{a}$ Light scattering measurements were not made on the sample, because precipitates were observed in the solution.

TABLE 2. Hydrodynamic Radius Evaluated from the Peaks in FIG. 2.

\begin{tabular}{lcc}
\hline Sample & $R_{\mathrm{h}, 1}{ }^{\mathrm{a}}$ & $R_{\mathrm{h}, 2}{ }^{\mathrm{b}}$ \\
\hline G1-0 & 213 & 3.6 \\
G1-10 & 104 & 5 \\
G1-20 & - & 13.2 \\
G2-0 & 160 & 6.7 \\
G2-10 & 109 & 7.4 \\
\hline
\end{tabular}

${ }^{\mathrm{a}}$ : Peak for the slow mode

b : Peak for the fast mode 
Figure captions

FIG. 1. Angular dependence of $K c / R_{\theta}$ for aqueous gelatin irradiated with 0 (filled circles), 10 (open circles) and $20 \mathrm{kGy}$ (filled triangles). (A) $c=5 \times 10^{-4}$, (B) $c=1 \times 10^{-3} \mathrm{~g} \mathrm{~cm}^{-3}$.

FIG. 2. Results of CONTIN analyses for aqueous gelatin solutions. Symbols denote the same meaning as Fig. 1. (A) $c=5 \times 10^{-4}$, (B) $c=1 \times 10^{-3} \mathrm{~g} \mathrm{~cm}^{-3}$.

FIG. 3. Reciprocal of scattering intensity for the fast mode for G1-10 (open circles), G1-20 (filled circles), and G2-10 (filled triangles). The solid lines are drawn for the guide of eyes. The tiny slope for G2-10 corresponds to $R_{\mathrm{g}}=$ $18 \mathrm{~nm}$.

FIG. 4. Circular dichroism spectra at the temperatures of 20 (squares), 30 (circles), and $40^{\circ} \mathrm{C}$ (triangles) for a fixed gelatin concentration of $1 \times 10^{-3} \mathrm{~g} \mathrm{~cm}^{-3}$. Irradiation dose is (A) 0 kGy, (B) $10 \mathrm{kGy}$, and (C) $20 \mathrm{kGy}$. 

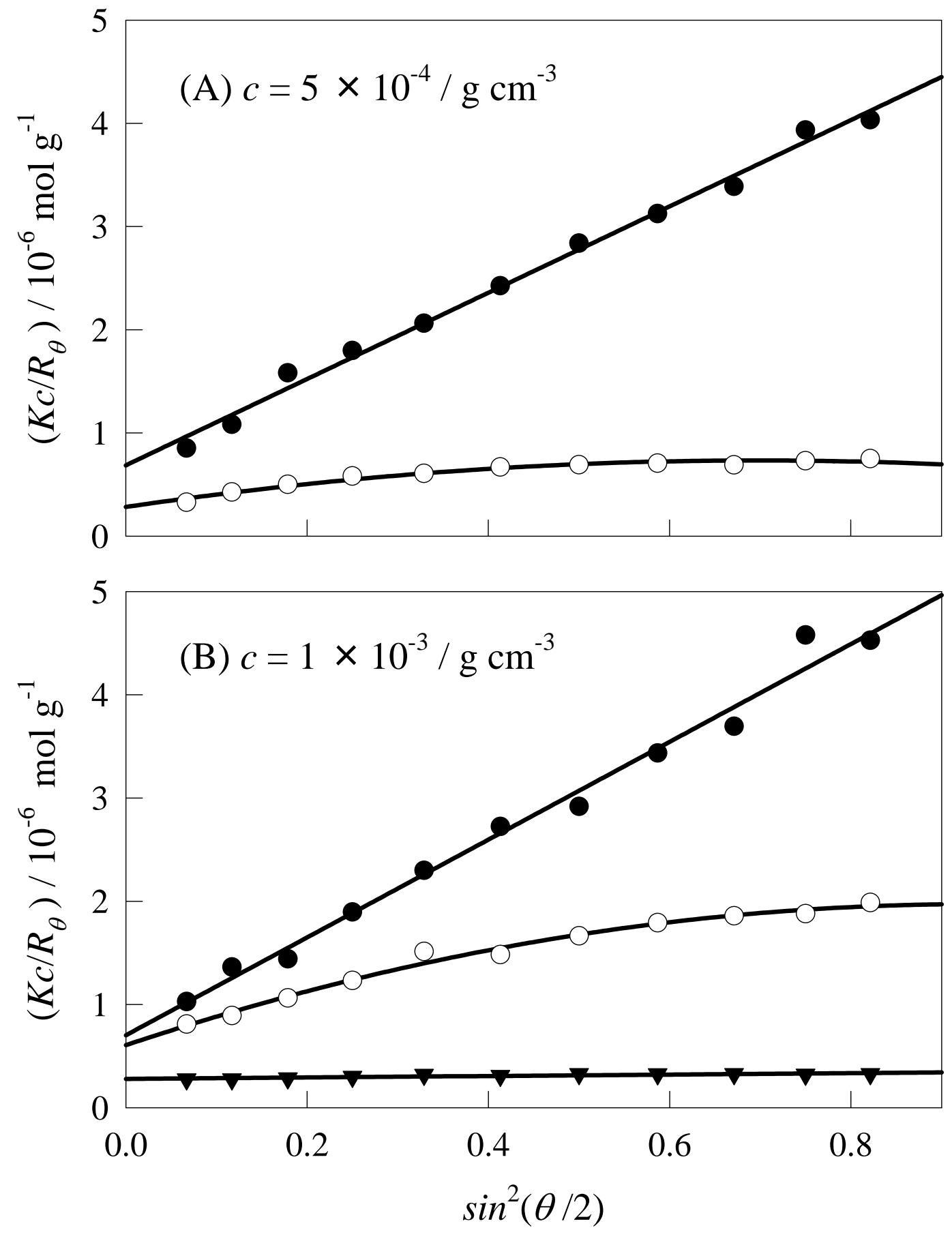

FIG. 1. Furusawa et al. 

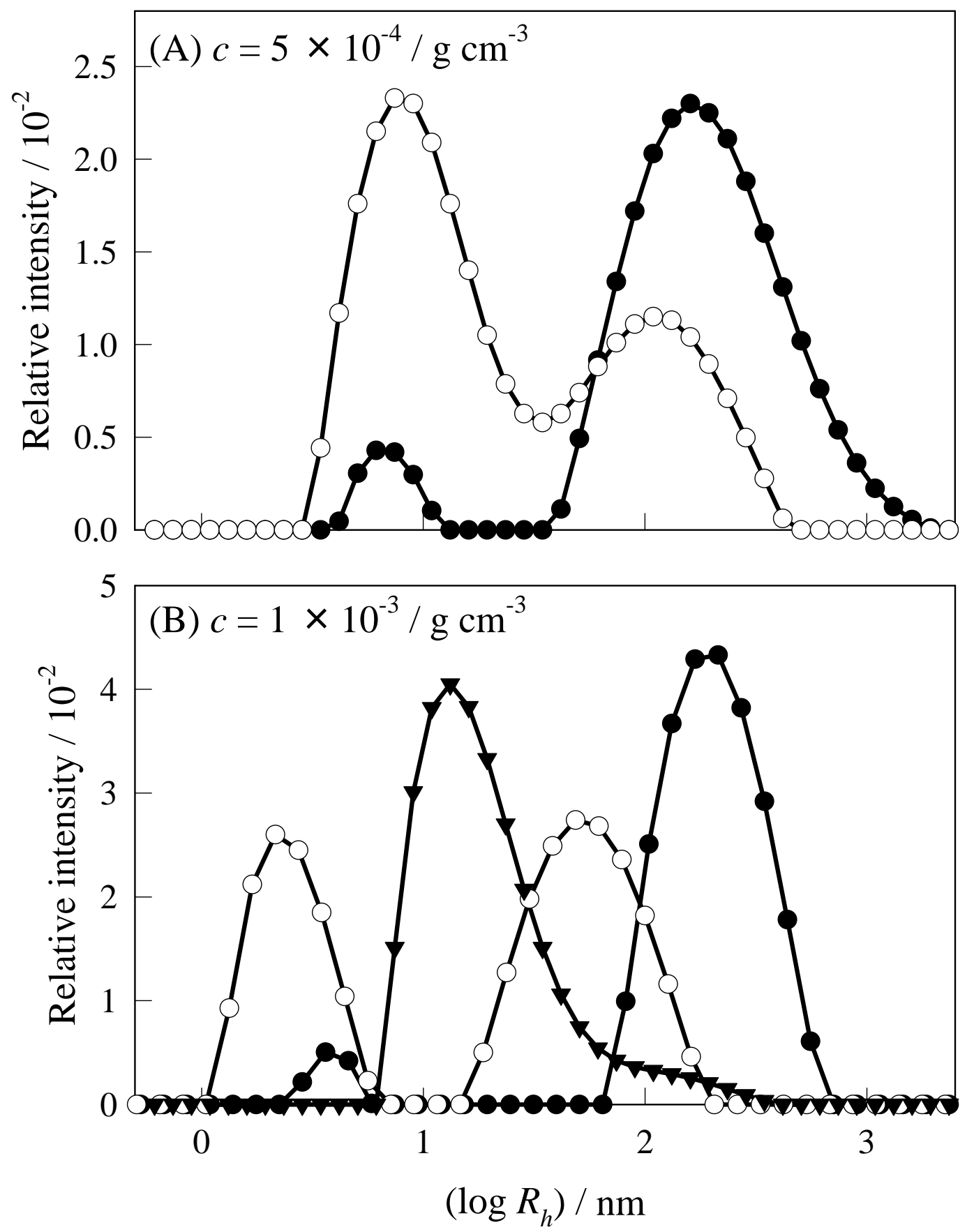

FIG. 2. Furusawa et al. 


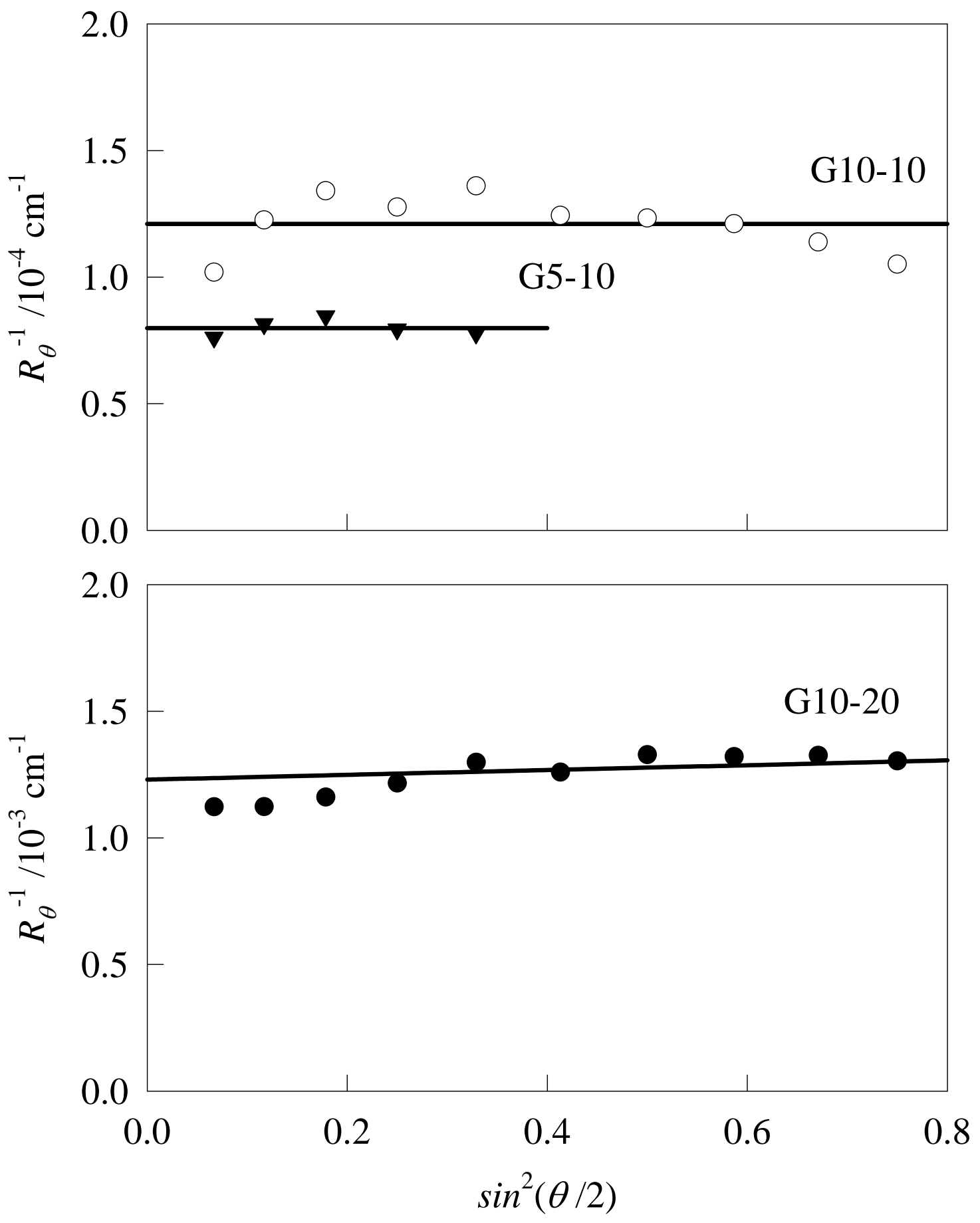

FIG. 3. Furusawa et al. 


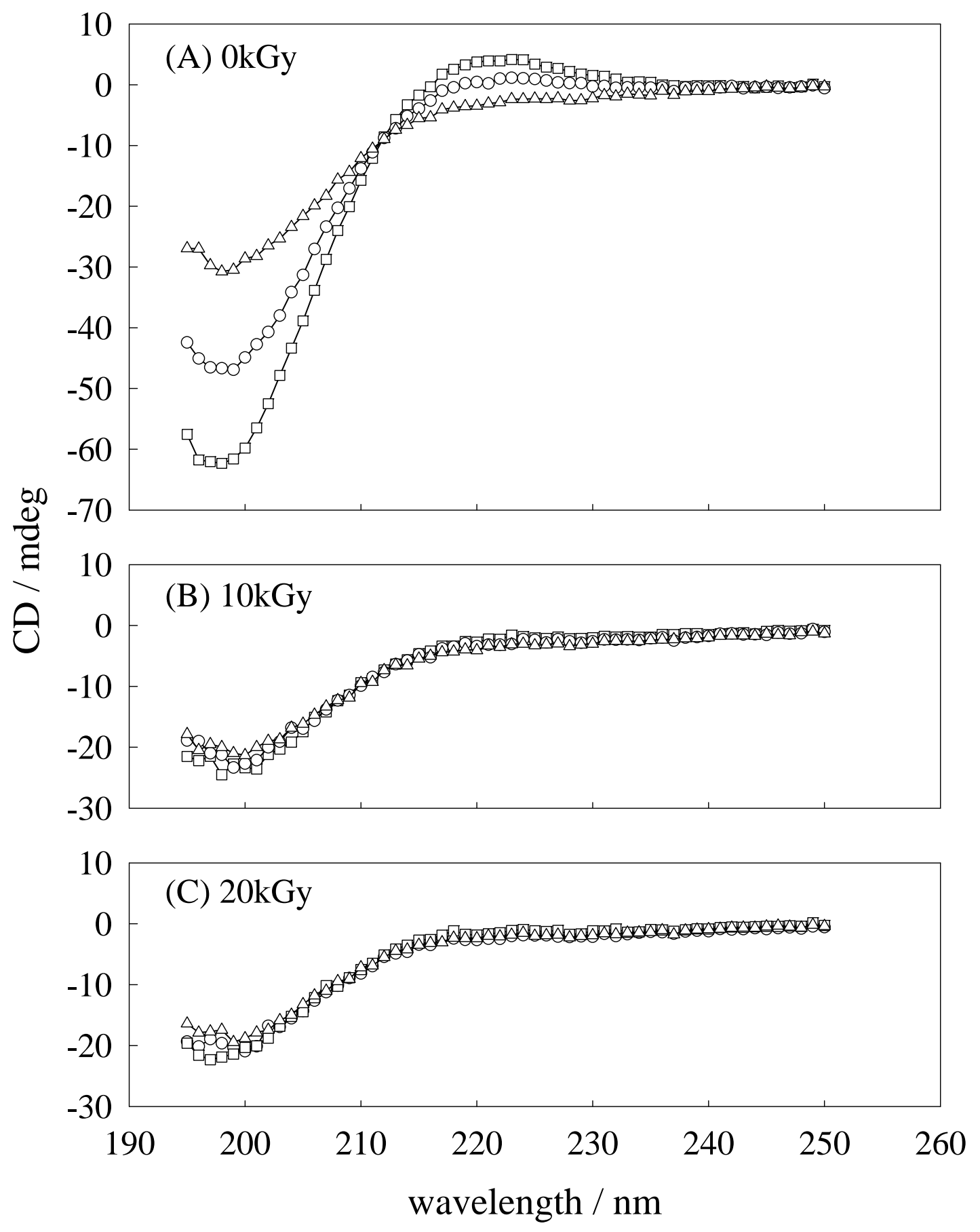

FIG. 4. Furusawa et al. 